\title{
ON THE FORMATION OF NARROW BUBBLE TRACKS BY A LASER BEAM IN ARGON, NITROGEN AND HYDROGEN BUBBLE CHAMBERS
}

\author{
G. HARIGEL, H.J. HILKE, G. LINSER and F. SCHENK \\ CERN, Geneva, Switzerland
}

Received 3 April 1981

Long bubble tracks, $<1 \mathrm{~mm}$ in diameter, have been produced in liquid argon and nitrogen with a nitrogen laser beam $(\lambda=$ $337 \mathrm{~nm}$ ), offering subnanosecond pulses with small beam divergence. Bubble formation was observed over a wide range of operating conditions, including those for ordinary bubble chamber operation, with a laser pulse of $10 \mu \mathrm{J}$. Typical bubble densities obtained were $4-8$ bubbles $/ \mathrm{cm}$, with maximum densities of 20 bubbles $/ \mathrm{cm}$. In liquid hydrogen, tracks of $3 \mathrm{~m}$ visible length were observed with track diameters from 1.5 to $6 \mathrm{~mm}$ and bubble densities from 2 to 25 bubbles/cm. Results and applications will be discussed.

\section{Introduction}

The basic aim of this work was to produce thin tracks in bubble chambers by short laser pulses. Such a technique could find various applications: production of fiducial lines, study of bubble growth for small bubbles, tagging of events and performance studies of rapid cyclicng and holographic bubble chamber.

Many years ago, the possibility was discussed of providing fiducial lines by a laser beam, especially in large bubble chambers [1], for monitoring optical distortions and bubble migration. At the other end of the scale, great progress in small bubble chambers with high resolution optics and holographic techniques has been prompted by the interest in lifetime measurements for short lived particles [2]. This work calls for better knowledge of bubble growth parameters for small diameters. $8 \mu \mathrm{m}$ bubbles have been photographed with only $7 \mu$ s flash delay but little is known about bubble growth rates for diameters below 40 $\mu \mathrm{m}$. Such studies require a well localized beam with small time jitter.

Another application could be foreseen in a large argon bubble chamber [3]. This liquid offers very interesting features: high electron mobility and efficient scintillation. If the scintillation pulse can be used for triggering, the laser beam - fired with very short delay - may provide a reference track to tag the event by producing the same bubble diameters.

Two groups have published results on bubble formation along laser beams in bubble chambers filled with $\mathrm{CF}_{3} \mathrm{Br}[4,5]$. It was assumed that bubble forma- tion was initiated on small dirt particles. Pulses of $30-600 \mu$ s duration and $20-200 \mathrm{~mJ}$ produced beam diameter of $\sim 10 \mathrm{~mm}$ and bubble densities of a few $10^{-2}$ bubbles $/ \mathrm{mm}^{3}$.

For the application we have in mind, beam diameters of $1 \mathrm{~mm}$ with $\sim 1$ bubble $/ \mathrm{mm}^{3}$ and much shorter pulse durations are required. A nitrogen laser was, therefore, used which had been developped to produce thin ionization tracks in gases with good time definition [6]. It delivers $0.1 \mathrm{~mJ}$ in $0.5 \mathrm{~ns}$ with beam cross section of $1 \times 2 \mathrm{~mm}^{2}$ and $0.8 \mathrm{mrad}$ divergence. We will describe the experimental arrangement and the results obtained in argon and nitrogen, with only $10 \mu \mathrm{J}$ delivered to the liquid.

\section{Experimental arrangement}

The experimental arrangement is shown in fig. 1. The 2.71 bubble chamber has a diameter of $16 \mathrm{~cm}$ and a height of $10 \mathrm{~cm} \mathrm{[3].} \mathrm{It} \mathrm{uses} \mathrm{bright} \mathrm{field} \mathrm{illumi-}$ nation via a Scotchlite disc of $11 \mathrm{~cm}$ diameter glued to the piston, which determines the visible track length. The temperature can be controlled to $\pm 0.1 \mathrm{~K}$, the static pressure and the pressure drop during expansion to \pm 0.05 and \pm 0.5 bar, respectively. The light pulses, produced by a two stage nitrogen laser $(\lambda=337 \mathrm{~nm})$, passes through two windows and is deflected by a front surface mirror in the vacuum tank and a stainless steel mirror in the liquid. Absorption and diffusion in the two windows and on the mirrors account for the low transmission of $\sim 10 \%$, as 


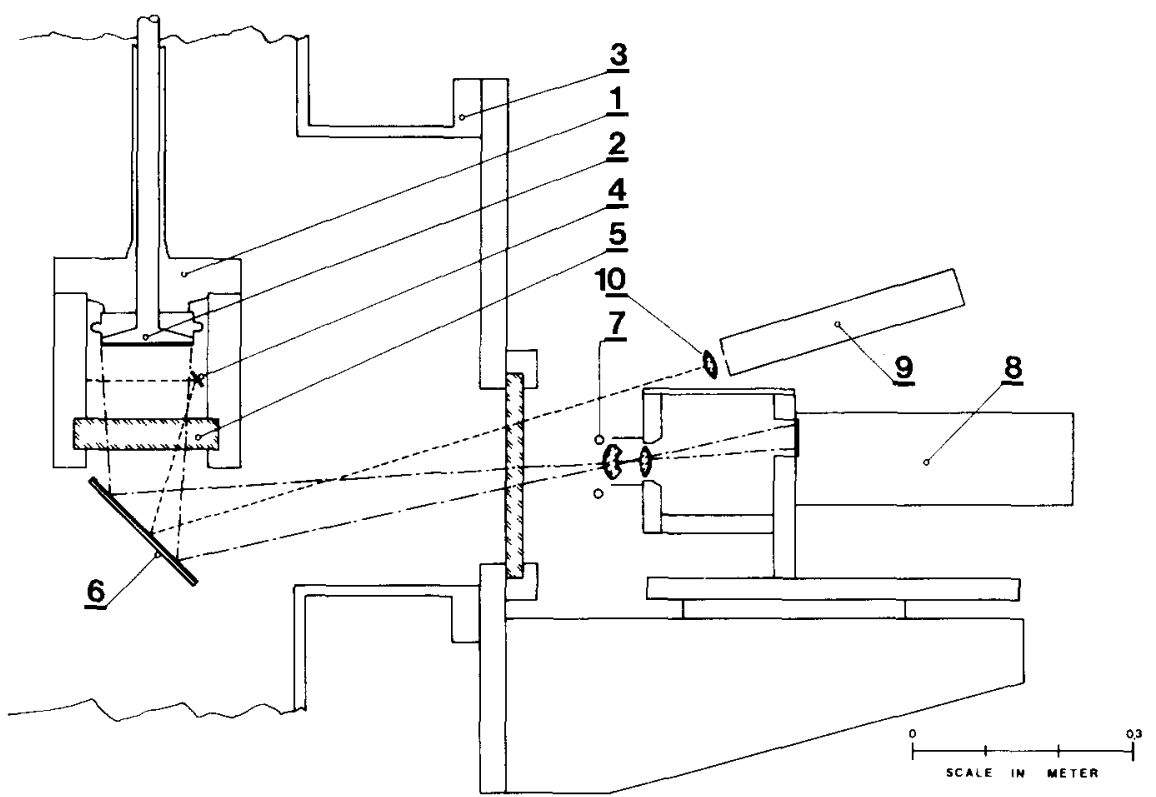

Fig. 1. Experimental arrangement. $1=$ bubble chamber body; $2=$ expansion piston with Scotchlite; $3=$ vacuum tank; $4=$ stainless steel mirror; $5=$ chamber window; $6=$ mirror; $7=$ lens with annular flash; $8=$ camera; $9=$ UV laser; $10=1$ ens.

measured by a bolometer. The intensity for bubble formation was, therefore, approximately $10 \mu \mathrm{J}$.

To vary the beam conditions, lenses with focal length of $0.5 \mathrm{~m}$ and $1.0 \mathrm{~m}$ and neutral filters were positioned in front of the laser. For other tests, a $1: 3$ beam expander was placed into the path between the two laser stages to decrease the beam divergence.

The argon and nitrogen was condensed into the chamber by cooling with liquid nitrogen. The gas impurities, as guaranteed by the supplier, were $<1 \mathrm{ppm}$ $\mathrm{H}_{2} \mathrm{O}, 0.3 \mathrm{ppm} \mathrm{N}_{2}, 0.1 \mathrm{ppm} \mathrm{O}_{2}$ for argon and $1 \mathrm{ppm}$ $\mathrm{H}_{2} \mathrm{O}, 0.5 \mathrm{ppm} \mathrm{O}_{2}$ for nitrogen.

\section{Results in argon and nitrogen}

Bubble formation was observed over wide temperature ranges: from 113.7 to $135.5 \mathrm{~K}$ in argon and 100.4 to $113.5 \mathrm{~K}$ in nitrogen (fig. 2). These ranges are wider than and including those for bubble formation by ionizing particles. Probably, laser induced bubble nucleation also occurs outside this temperature range, especially at lower temperatures.

With 2 ms flash delay, bubble diameters are similar to those obtained with ionising particles during earlier tests [3] and those theoretically expected for the lower temperature range. Maximum bubble diameters were $0.4 \mathrm{~mm}$ in argon and $0.7 \mathrm{~mm}$ in nitrogen. The track diameter-defined as containing $2 / 3$ of the bubbles in the visible projection - was varied with lenses and a beam expander. The unfocused laser beam produced track diameters of $2.4 \mathrm{~mm}$, with a

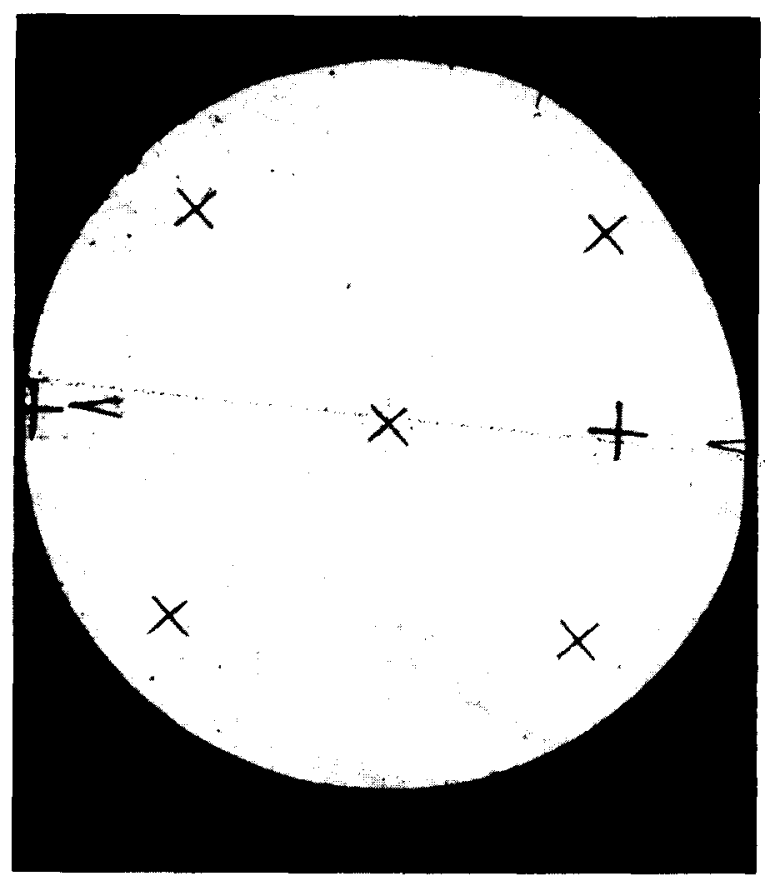

Fig. 2. Photo of laser induced track in nitrogen. Chamber conditions: $T=100.4 \mathrm{~K}, p_{\min }=3.5 \mathrm{bar}$, flash delay $=2 \mathrm{~ms}$. 
$1.1 \mathrm{~m}$ distance between laser and bubble chamber liquid. This track diameter was reduced to $1.2 \mathrm{~mm}$ by focusing the light at $0.5 \mathrm{~m}$ in front of the liquid (lens with focal length $f=0.5 \mathrm{~m}$ ), and to $0.6 \mathrm{~mm}$ by focusing just in front of the chamber entrance window (with $f=1.0 \mathrm{~m}$ ). The introduction of the $1: 3$ beam expander again lead to a beam diameter of $\sim 0.6 \mathrm{~mm}$, but with lower bubble densities.

These measurements demonstrate, that it is possible to create track diameters of $\sim 1 \mathrm{~mm}$ over a length of $1 \mathrm{~m}$.

The bubble densities were typically 4 bubbles $/ \mathrm{cm}$ in argon and 8 bubbles $/ \mathrm{cm}$ in nitrogen, maximum densities 8 bubbles $/ \mathrm{cm}$ in argon and 20 bubbles $/ \mathrm{cm}$ in nitrogen.

A crude estimate of the absorption of the laser intensity in the liquid was obtained by measuring bubble densities in the first and last third of many tracks, i.e. at beam entry and beam exit. From the ratio of these counts we conclude that the absorption is $<5 \%$ over $7.5 \mathrm{~cm}$.

The dependence of bubble densities on laser intensity was measured by the insertion of neutral density filters. The average number of bubbles per track $(11 \mathrm{~cm})$ in argon at $122.6 \mathrm{~K}$ were: $42,5.6$ and $<0.5$ for filter transmission of 100,50 and $20 \%$, respectively. The dependence is, therefore, stronger than quadratic, almost cubic over this range.

In addition to the bubble track in the liquid, relatively strong bubble formation was observed on the bubble chamber window as well as on the Scotchlite, if the beam was moved to impinge on the latter. The diameter of these bubble clouds was twice as large as the diameter of the track in the liquid.

\section{Results in hydrogen}

In the Big European Bubble Chamber (BEBC), tracks of $3 \mathrm{~m}$ visible length were observed at $26 \mathrm{~K}$, under normal operating conditions: static pressure $=$ 5.3 bar, pressure drop $=3.8$ bar, flash delay $=10 \mathrm{~ms}$. With 2-5 bubbles $/ \mathrm{cm}$ the tracks showed diameters of less than $2 \mathrm{~mm}$, with $15-25$ bubbles/cm diameters from 3.5 to $6 \mathrm{~mm}$. The maximum laser energy transmitted to the liquid was $100 \mu \mathrm{J}$.

\section{Discussion of the results}

It is interesting to look into the bubble nucleation process in some detail. Bubble formation by ionizing particles proceeds basically in two steps: at first the liquid is heated locally by $\delta$-electrons sufficiently to produce a bubble of radius greater than a critical value in a very short time $(\leqslant 1 \mathrm{~ns})$. The critical radius is determined by a balance of forces between the surface tension and the pressure difference between the vapour in the bubble and the liquid. Above the critical radius, the surface tension diminishes rapidly, and heat inflow from the surrounding liquid alone will sustain further bubble growth.

This second stage in the bubble growth should be identical for bubbles initiated by a laser beam. The first nucleation phase, however, most likely requires the presence of inhomogeneities like tiny solid absorption centres or solid surfaces [7]. This may be deduced from the following estimates. We will choose argon at $T_{0}=134 \mathrm{~K}$ with an expansion $p_{\text {vapour }}\left(T_{0}\right)-$ $p_{\min }=13.5$ bar for our calculations.

The critical radius $r_{\mathrm{c}}$ is given by $r_{\mathrm{c}}=2 \sigma / \Delta p=75 \AA$ for a surface tension $\sigma=5 \times 10^{-3} \mathrm{~N} / \mathrm{m}$. The minimum energy required to form a bubble with critical radius is [8]

$E_{\mathrm{c}}=4 \pi r_{\mathrm{c}}^{2}(\sigma-T \mathrm{~d} \sigma / \mathrm{d} T)+\frac{4}{3} \pi r_{\mathrm{c}}^{3}\left(\rho^{\prime} h+p_{\min }\right)$,

where $T=$ temperature $=134 \mathrm{~K}, \quad \rho^{\prime}=$ density of vapour $=0.14 \mathrm{~g} / \mathrm{cm}^{3}, h=$ heat of vaporization $=$ $90 \mathrm{~J} / \mathrm{g}$. One obtains

$E_{\mathrm{c}}=230 \mathrm{eV}$.

This energy at least has to be provided to the liquid in a time not much longer than the time $t_{\mathrm{D}}$, in which heat diffuses out of the critical bubble

$t_{\mathrm{D}}=r_{\mathrm{c}}^{2} \rho c_{\mathrm{p}} / 4 \lambda \lesssim 0.4 \mathrm{~ns}$,

with density of liquid $\rho=1.01 \mathrm{~g} / \mathrm{cm}^{3}$, specific heat $c_{\mathrm{p}}$ assumed as $2 \mathrm{~J} / \mathrm{g} \cdot \mathrm{K}$ and heat conductivity of the liquid $\lambda=8 \times 10^{-4} \mathrm{~W} / \mathrm{K} \cdot \mathrm{cm}$.

The laser pulse contains $\sim 2 \times 10^{13}$ photons of 3.7 $\mathrm{eV}$ each, in a beam diameter of $\sim 1 \mathrm{~mm}$. If $230 \mathrm{eV}$ would be absorbed directly in the liquid in a sphere of critical radius $\left(V \sim 2 \times 10^{-15} \mathrm{~mm}^{3}\right), 4 \times 10^{16}$ photons would be absorbed in $1 \mathrm{~mm}$ track length $(V \sim$ $1 \mathrm{~mm}^{3}$ ). With a measured absorption length of the order of a metre, this process can be excluded for bubble nucleation, even under the assumption of very large fluctuations in the energy absorption.

On the other hand, the presence of microparticles with strong absorption may explain the bubble formation [7]. From the measurement of approximately 10 bubbles $/ \mathrm{cm}$ in a beam with $1 \mathrm{~mm}$ diameter, it follows that more than $10^{3}$ absorber centres $/ \mathrm{cm}^{3}$ are 
required. With an impurity level of $1 \mathrm{ppm}$ this leads to an average impurity diameter $<10 \mu \mathrm{m}$. A black body of $10 \mu \mathrm{m}$ diameter would absorb some $10^{10} \mathrm{eV}$ from the laser beam $\left(10^{14} \mathrm{eV}\right.$ in $\left.\emptyset=1 \mathrm{~mm}\right)$. Any process with an efficiency of $\gtrsim 10^{-7}$ for converting this energy into evaporization of a critical bubble could, therefore, account for the observed bubble formation.

Short laser pulses - of the order of the heat diffusion time $t_{\mathrm{D}}=0.5 \mathrm{~ns}-$ are probably essential for efficient energy transmission to the bubble. This may explain at least partially, that a much lower pulse energy $(10 \mu \mathrm{J})$ was required for bubble formation than in the earlier experiments $(20-200 \mathrm{~mJ}$ producing a two orders of magnitude lower bubble density). These earlier experiments used freon, but this should not introduce a big difference, as the bubble formation energy is similar: $150 \mathrm{eV}$. In ref. 5 it was shown that the bubble formation was independent of the wavelength $\lambda$ for $\lambda>400 \mathrm{~nm}$. The lower wavelength used in our study should, therefore, not forbid a direct comparison.

\section{Conclusions and outlook}

The formation of long, thin bubble tracks in liquid argon, formed nitrogen and hydrogen promises to find various applications. As the bubbles are probably on tiny impurities, it is expected that similar results will be found in other liquids. In liquid argon various further studies are planned: comparison of simultaneous bubble formation by ionizing tracks and by laser beams; the production of electrons in the liquid (probably on the impurities) by the laser beam using a charge measuring device; and tagging of particle interactions using the scintillation signal from the interaction as trigger.

The authors are greatly indebted to many people in the BEBC group at CERN for their dedicated help during construction and tests of the bubble chamber, in particular to J. Armand, W. Bichler, A. Carrere, J. Feyt, E. Murer, F. Pouyat, P. Rada, E. Rusconi, G. Schmidlin, W. Seidl, D. Voillat and W. Wilkens as well as to M. Brunet, Dr. G. Kuhn and R. Schuler for their skilful work related to the nitrogen laser. Continuous support for the project from Drs. A. Minten, P. Lazeyras, H.P. Reinhard and H. Wenninger is gratefully acknowledged.

\section{References}

[1] See, for example, (a) R.K. Böck, H. Leutz, H. Wenninger and J. Zoll, Internal report CERN/D.Ph.II./FPC 68-6 (1968), (b) G. Harigel, Internal note CERN/BEBC/OP-19 (1970).

[2] M. Dykes, P. Lecoq, D. Güsewell, A. Hervé, H. Wenninger, H. Royer, B. Hahn, E. Hugentobler, E. Ramseyer and M. Boratav, Nucl. Instr. and Meth. 179 (1981) 487.

[3] G. Harigel, G. Linser and F. Schenk, submitted to Nucl. Instr. and Meth. (1981).

[4] R.C. Stamberg and D.E. Gillespie, j. Appl. Phys. 37 (1966) 459.

[5] F.H. Poesposoetjipto and E. Hugentobler, Helv. Phys. Acta 43 (1970) 203.

[6] H.J. Hilke, Nucl. Instr. and Meth. 174 (1980) 145.

[7] The same conclusion was already put forward by Askar'yan et al. who had observed bubble formation by a laser beam in water and by refs. 4, 5; G.A. Askar'yan, A.M. Prokorov, G.F. Chanturiya and G.P. Shipuro, Sov. Phys. JETP 17 (1963) 1463.

[8] D.V. Bugg, Progr. Nucl. Phys. 7 (1959). 